\title{
DYNAMICS OF LAMINATED COMPOSITE PLATES WITH A HIGHER ORDER THEORY AND FINITE ELEMENT DISCRETIZATION
}

\author{
Mallikarjuna AND T. Kant \\ Department of Civil Engineering, Indian Institute of Technology, \\ Powai, Bombay 400 076, India
}

(Received 7 October 1987, and in revised form 22 April 1988)

\begin{abstract}
A simple isoparametric finite element formulation based on a higher-order displacement model for dynamic analysis of multi-layer symmetric composite plates is presented with an explicit time marching scheme. As is well-known, the Kirchhoff plate theory is inadequate to describe the propagation of waves in plates. The first-order shear deformable Mindlin plate theory hitherto considered was adequate for determining responses to dynamic loads. The present higher-order theory which is more accurate than the Mindlin theory is applied, herein, for the evaluation of plate response to different types of dynamic loads. A special mass lumping scheme is adopted which conserves the total mass of the element and includes the effects due to rotary inertia terms. The parametric effects of the time step, finite element mesh, lamination scheme and orthotropy on the transient response are investigated. Several numerical examples are presented and compared with results from other sources.
\end{abstract}

\section{INTRODUCTION}

In recent years composite materials have been widely used, largely because of their superior mechanical properties such as combination of high stiffness and high strength with low density and potentially low unit cost. There exists a need for assessing the transient response of laminated plates. In this study emphasis is placed on establishing the credibility of a higher order shear deformable (or penalty) finite element for transient analyses, and in obtaining transient solutions to general composite plate problems. Apparently, this study constitutes the first in which the transient response of composite plates of arbitrary construction and finite dimensions has been analyzed by using a higher order theory.

The following brief review of the literature on transient response of elastic plates provides the necessary background. The theory of thin laminated composite plates has been established by Lekhnitskii [1], Reissner and Stavsky [2] Dong, Pister and Taylor [3], and Stavsky [4]. Reismann and his colleagues [5-7] analyzed a simply supported, rectangular, isotropic plate subjected to a suddenly applied uniformly distributed load over a square area of the plate. Rock and Hinton [8] presented a transient finite element analysis of thick and thin isotropic plates. Excellent agreement of the finite element solutions with the analytical solutions of Reismann and Lee [5] was obtained. Akay [9] determined the large deflection transient response of isotropic plates using a mixed finite element. All of these studies were confined to homogeneous, isotropic plates.

Moon $[10,11]$ investigated the response of infinite laminated plates subjected to transverse impact loads at the centre of the plate. Chow [12] employed the Laplace transform technique to investigate the dynamic response of orthotropic laminated plates. 
Wang, Chou and Rose [13] applied the method of characteristics to unsymmetrical orthotropic laminated plates. Sun and his colleagues [14-17] employed the classical method of separation of variables combined with the Mindlin-Goodman [18] procedure for treating time-dependent boundary conditions and/or dynamic external loadings. These papers dealt with plates under cylindrical bending. Recently, Reddy [19,20] presented closed form and finite element results for the transient analysis of layered composite plates. All of these investigations were based on either the classical (Kirchhoff) plate theory or the first order shear deformable (Mindlin-Reissner) theory.

Theories based on realistic displacement models which give rise to non-linear distribution of in-plane normal strains and transverse shear strains have been developed by Murthy [21], and Phan and Reddy [22]. In these works, although the zero transverse shear stress conditions on the top and bottom plate surfaces are satisfied, the approaches involve the computationally inefficient $C^{1}$ finite element formulations. Lo, Christensen and $\mathrm{Wu}$ [23] and Kant [24] have, in addition, included the effects of transverse normal strain and stress in their theories. Kant, Owen and Zienkiewicz [25] have presented a $C^{0}$ finite element formulation of the higher order theory. Kant and Pandya [26-31] have extended the above formulation for generally orthotropic plates. All of these papers were confined to static analyses.

This paper specifically deals with the application of a $C^{0}$ isoparametric finite element for transient analysis of multi-layer symmetrically laminated composite plates based on a higher order displacement model theory. The bi-quadratic Lagrangian nine noded quadrilateral element is used together with the selective integration technique for the numerical computations.

\section{THEORY}

The present higher order shear deformable theory for symmetrically laminated plates is developed by assuming the displacement field in the following form:

$$
\begin{gathered}
u(x, y, z, t)=z \theta_{x}(x, y, t)+z^{3} \theta_{x}^{*}(x, y, t), \\
v(x, y, z, t)=z \theta_{y}(x, y, t)+z^{3} \theta_{y}^{*}(x, y, t), \quad w(x, y, z, t)=w_{0}(x, y, t),
\end{gathered}
$$

in which $t$ is the time, $w_{0}$ represents the transverse displacement of the midplane and $\theta_{x}$, $\theta_{y}$ are the rotations of the normals to the midplane about the $y$ and $x$ axes respectively. The parameters $\theta_{x}^{*}, \theta_{y}^{*}$ are the higher order terms which account for the flexural mode of deformation in the Taylor's series expansion and are also defined at the midplane.

By substitution of relations (1) in the strain displacement equations of the classical theory of elasticity, the following relationships are obtained:

$$
\left[\begin{array}{c:c}
\varepsilon_{x} & \gamma_{x y} \\
\varepsilon_{y} & \gamma_{y z} \\
\varepsilon_{z} & \gamma_{x z}
\end{array}\right]=\left[\begin{array}{c:c}
z K_{x}+z^{3} K_{x}^{*} & z K_{x y}+z^{3} K_{x y}^{*} \\
z K_{y}+z^{3} K_{y}^{*} & \Phi_{y}+z^{2} \Phi_{y}^{*} \\
0 & \Phi_{x}+z^{2} \Phi_{x}^{*}
\end{array}\right]
$$

Here,

$$
\begin{gathered}
{\left[K_{x}, K_{y}, K_{x y}\right]^{\mathrm{t}}=\left[\partial \theta_{x} / \partial x, \partial \theta_{y} / \partial y, \partial \theta_{x} / \partial y+\partial \theta_{y} / \partial x\right]^{\mathrm{t}}} \\
{\left[K_{x}^{*}, K_{y}^{*}, K_{x y}^{*}\right]^{\mathrm{t}}=\left[\partial \theta_{x}^{*} / \partial x, \partial \theta_{y}^{*} / \partial y, \partial \theta_{x}^{*} / \partial y+\partial \theta_{y}^{*} / \partial x\right]^{\mathrm{t}},} \\
{\left[\Phi_{x}, \Phi_{y}, \Phi_{x}^{*}, \Phi_{y}^{*}\right]^{\mathrm{t}}=\left[\partial w_{0} / \partial x+\theta_{x}, \partial w_{0} / \partial y+\theta_{y}, 3 \theta_{x}^{*}, 3 \theta_{y}^{*}\right]^{\mathrm{t}},}
\end{gathered}
$$

where $t$ denotes the transpose. The stress-strain relations for the $L$ th lamina in the laminate 
co-ordinate axes $(x-y-z$ - (see Figure 1$)$ are written as

$$
\left\{\begin{array}{c}
\sigma_{x} \\
\sigma_{y} \\
\tau_{x y}
\end{array}\right\}^{L}\left[\begin{array}{lll}
Q_{11} & Q_{12} & Q_{13} \\
Q_{12} & Q_{22} & Q_{23} \\
Q_{13} & Q_{23} & Q_{33}
\end{array}\right]^{L}\left\{\begin{array}{c}
\varepsilon_{x} \\
\varepsilon_{y} \\
\gamma_{x y}
\end{array}\right\}^{L}, \quad\left\{\begin{array}{c}
\tau_{y z} \\
\tau_{x z}
\end{array}\right\}^{L}=\left[\begin{array}{ll}
Q_{44} & Q_{45} \\
Q_{45} & Q_{55}
\end{array}\right\}^{L}\left\{\begin{array}{l}
\gamma_{y z} \\
\gamma_{x z}
\end{array}\right\}^{L}, \quad(3 \mathrm{a}, \mathrm{b})
$$

where

$$
\sigma=\left\{\sigma_{x}, \sigma_{y}, \tau_{x y}, \tau_{y z}, \tau_{x z}\right\}^{\mathrm{t}}, \quad \varepsilon=\left\{\varepsilon_{x}, \varepsilon_{y}, \gamma_{x y}, \gamma_{y z}, \tau_{x z}\right\}^{\mathrm{t}}
$$

are the stress and linear strain vectors with reference to the laminate axes and $Q_{i j}$ 's are the transformed reduced elastic coefficients in the plate (laminate) axes of the $L$ th lamina. The transformation of the stresses/strains between the lamina and the laminate coordinate systems follows the usual transformation rule [32].

The constitutive relations involving bending moments and shear forces are defined as follows:

$$
\begin{aligned}
& {\left[\begin{array}{c:c}
M_{x} & M_{x}^{*} \\
M_{y} & M_{y}^{*} \\
M_{x y} & M_{x y}^{*}
\end{array}\right]=\sum_{L=1}^{n} \int_{h_{L}}^{h_{L+1}}\left\{\begin{array}{c}
\sigma_{x} \\
\sigma_{y} \\
\tau_{x y}
\end{array}\right\}\left[z: z^{3}\right] \mathrm{d} z,} \\
& {\left[\begin{array}{ll}
Q_{x} & Q_{x}^{*} \\
Q_{y} & Q_{y}^{*}
\end{array}\right]=\sum_{L=1}^{n} \int_{h_{L}}^{h_{L+1}}\left\{\begin{array}{l}
\tau_{x z} \\
\tau_{y z}
\end{array}\right\}\left[\begin{array}{lll} 
& z^{2}
\end{array}\right] \mathrm{d} z .}
\end{aligned}
$$

After integration, these relations are written in a matrix form which defines the stress-resultant/strain relations of the laminate:

$$
\left\{\begin{array}{c}
\mathbf{M} \\
\mathbf{M}^{*} \\
\mathbf{Q} \\
\mathbf{Q}^{*}
\end{array}\right\}=\left[\begin{array}{c:c}
D_{b} & 0 \\
\hdashline 0 & D_{s}
\end{array}\right]\left\{\begin{array}{c}
\mathbf{K} \\
\mathbf{K}^{*} \\
\mathbf{\Phi} \\
\mathbf{\Phi}^{*}
\end{array}\right\}, \quad \text { or } \quad \tilde{\mathbf{\sigma}}=\underline{D} \overline{\boldsymbol{\varepsilon}}
$$

Here

$$
\begin{array}{cc}
\mathbf{M}=\left\{M_{x}, M_{y}, M_{x y}\right\}^{\mathrm{t}}, & \mathbf{K}=\left\{K_{x}, K_{y}, K_{x y}\right\}^{\mathrm{t},} \\
\mathbf{M}^{*}=\left\{M_{x}^{*}, M_{y}^{*}, M_{x y}^{*}\right\}^{\mathrm{t}}, & \mathbf{K}^{*}=\left\{K_{x}^{*}, K_{y}^{*}, K_{x y}^{*}\right\}^{\mathrm{t}}, \\
\mathbf{Q}=\left\{Q_{x}, Q_{y}\right\}^{\mathrm{t}}, \quad \boldsymbol{\Phi}=\left\{\Phi_{x}, \Phi_{y}\right\}^{\mathrm{t}}, \quad \mathbf{Q}^{*}=\left\{Q_{x}^{*}, Q_{y}^{*}\right\}^{\mathrm{t}}, \quad \boldsymbol{\Phi}^{*}=\left\{\Phi_{x}^{*}, \Phi_{y}^{*}\right\}^{\mathrm{t}},
\end{array}
$$

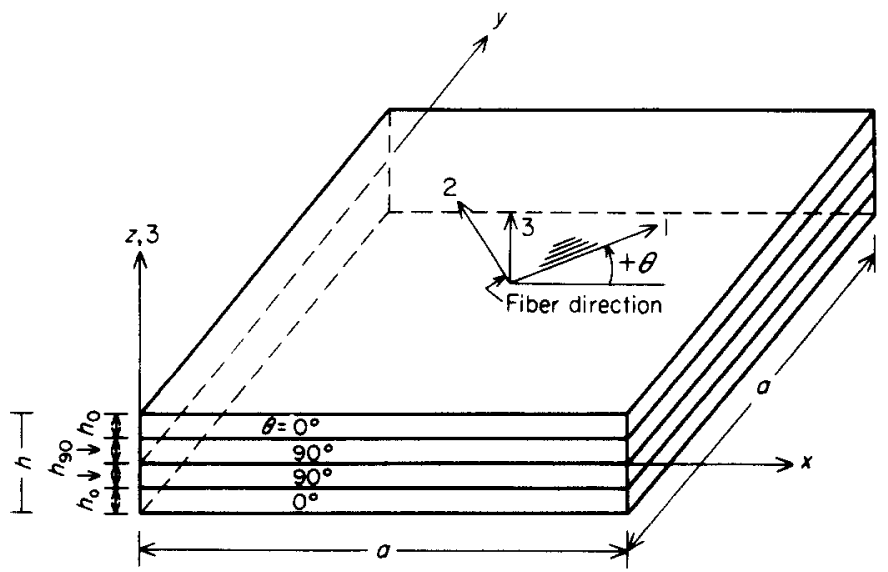

Figure 1. Laminate geometry with positive set of lamina/laminate reference axes, displacement components, and fibre orientation. 


$$
\begin{gathered}
D_{b}=\sum_{L=1}^{n}\left[\begin{array}{cccccc}
Q_{11} H_{3} & Q_{12} H_{3} & Q_{13} H_{3} & Q_{11} H_{5} & Q_{12} H_{5} & Q_{13} H_{5} \\
& Q_{22} H_{3} & Q_{23} H_{3} & Q_{12} H_{5} & Q_{22} H_{5} & Q_{23} H_{5} \\
& Q_{33} H_{3} & Q_{13} H_{5} & Q_{23} H_{5} & Q_{33} H_{5} \\
& & Q_{11} H_{7} & Q_{12} H_{7} & Q_{13} H_{7} \\
& & & Q_{22} H_{7} & Q_{23} H_{7} \\
\text { symmetric layer } & & & & Q_{33} H_{7}
\end{array}\right] \\
D_{s}=\sum_{L=1}^{n}\left[\begin{array}{ccccc}
Q_{55} H_{1} & Q_{45} H_{1} & Q_{55} H_{3} & Q_{45} H_{3} \\
& Q_{44} H_{1} & Q_{45} H_{3} & Q_{44} H_{3} \\
& & Q_{55} H_{5} & Q_{45} H_{5} \\
& & & Q_{44} H_{5}
\end{array}\right]^{\text {th layer }}
\end{gathered}
$$

In these relationships, $n$ defines the number of layers and

$$
H_{i}=(1 / i)\left(h_{L+1}^{i}-h_{L}^{i}\right), \quad i=1,3,5,7 \text {. }
$$

\subsection{FINITE ELEMENT DISCRETIZATION}

Finite element spatial discretization schemes, when applied to dynamic transient structural analysis problems, result in a set of ordinary differential equations. In the absence of damping these equations take the form

$$
M \ddot{\mathbf{a}}(t)+\underline{K} \mathbf{a}(t)=\mathbf{P}(t),
$$

in which the dots define differentiation with time $t, \mathbf{a}(t)$ is the nodal displacement vector, $M$ is the mass matrix and $\mathbf{P}(t)$ is the vector of forces which varies with time, $t$.

In $C^{\circ}$ finite element theory, the continuum displacement vector within the element is discretized such that

$$
\mathbf{a}(t)=\sum_{i=1}^{N E} N_{i}(x, y) \mathbf{a}_{i}(t)
$$

in which the term $N_{i}(x, y)$ is the interpolating (shape) function associated with node $i$, $a_{i}(t)$ is the value of $\mathbf{a}(t)$ corresponding to node $i$ and $N E$ is the number of nodes in the element.

Knowing the generalized displacement vector $\mathbf{a}(t)$ at all points within the element, one can express the generalized strain vector $\varepsilon$ at any point as

$$
\varepsilon=\sum_{i=1}^{N E}\left[\begin{array}{ccccc}
0 & \partial N_{i} / \partial x & 0 & 0 & 0 \\
0 & 0 & \partial N_{i} / \partial y & 0 & 0 \\
0 & \partial N_{i} / \partial y & \partial N_{i} / \partial x & 0 & 0 \\
0 & 0 & 0 & \partial N_{i} / \partial x & 0 \\
0 & 0 & 0 & 0 & \partial N_{i} / \partial y \\
0 & 0 & 0 & \partial N_{i} / \partial y & \partial N_{i} / \partial x \\
\partial N_{i} / \partial x & N_{i} & 0 & 0 & 0 \\
\partial N_{i} / \partial y & 0 & N_{i} & 0 & 0 \\
0 & 0 & 0 & 3 N_{i} & 0 \\
0 & 0 & 0 & 0 & 3 N_{i}
\end{array}\right]\left\{\begin{array}{c}
w_{i} \\
\theta_{x i} \\
\theta_{y i} \\
\theta_{x i}^{*} \\
\theta_{y i}^{*}
\end{array}\right],
$$

or

$$
\varepsilon=\sum_{i=1}^{N E} B_{i} \mathbf{a}_{i}(t)
$$


where

$$
\varepsilon=\left\{K_{x}, K_{y}, K_{x y}, K_{x}^{*}, K_{y}^{*}, K_{x y}^{*}, \Phi_{x}, \Phi_{y}, \Phi_{x}^{*}, \Phi_{y}^{*}\right\}^{\mathrm{t}}
$$

The computation of the element stiffness matrix is economized by explicit multiplication of the $\underline{B}_{i}, \underline{D}$ and $\underline{B}_{j}$ matrices instead of carrying out the full matrix multiplication of the triple product. Due to the symmetry of the stiffness matrix, only blocks lying on one side of the main diagonal are formed [33].

The mass matrix, $M$, in equation (11) is given by

$$
M=\int_{\text {Area }} \underline{N}^{\prime} \underline{\underline{m}} \underline{N} \mathrm{~d}(\text { Area })
$$

where

$$
\bar{m}=\left[\begin{array}{lllll}
I_{1} & & & & 0 \\
& I_{2} & & & \\
& & I_{2} & & \\
0 & & & I_{3} & \\
& & & & I_{3}
\end{array}\right],
$$

in which $I_{1}, I_{2}$ and $I_{3}$ are normal inertia, rotary inertia and higher order inertia terms respectively. These are defined as

$$
\left[I_{1}, I_{2}, I_{3}\right]=\sum_{L=1}^{n} \int_{h_{L}}^{h_{L+1}}\left[1, Z^{2}, Z^{6}\right] \rho^{L} \mathrm{~d} z
$$

where $\rho^{L}$ is the material density of the $L$ th layer.

The ordinary differential equation (11) is solved by using an explicit central difference scheme. This scheme can be written as

$$
\mathbf{a}^{n+1}=M^{-1}(\Delta t)^{2}\left(-\underline{K} \mathbf{a}^{n}+\mathbf{P}^{n}\right)-a^{n-1}+2 \mathbf{a}^{n},
$$

where superscripts $n-1, n, n+1$ denote three successive time stages and $\Delta t$ is the time step length. The main advantage of this approach is that if $M$ is diagonal, the computation at each time step is trivial. Unfortunately, for the parabolic isoparametric element used in the spatial discretization, $M$ is not diagonal and some mass lumping scheme must be used. A special mass diagonalization due to Hinton et al. [34] has been used here. For clarity, this can be stated as follows: (1) the diagonal coefficients of the consistant mass matrix $M_{i i}^{e}=\int_{A} N^{\top} \underline{\bar{m}} \underline{N} \mathrm{~d} A$ is computed; (2) the total mass of the element, $M^{e}=\int_{V} \rho \mathrm{d} V=$ $\int_{A} \rho t \mathrm{~d} A$, is also computed; (3) a number, $S$, is formed by adding the diagonal coefficients $M_{i i}^{e}$ associated with translation (but not rotation); (4) the diagonal coefficients of the mass matrix, $M_{i i}^{e}$ are scaled by multiplying them by the ratio $M^{e} / S$, thus preserving the total mass of the element.

After the mass matrix has been diagonalized the equation (15a) can be written as

$$
\mathbf{a}_{i}^{n+1}=\left[(\Delta t)^{2} / M_{i i}\right]\left\{-\sum_{j=1}^{N E} \underline{K}_{i j} \mathbf{a}_{j}^{n}+\mathbf{P}_{i}^{n}\right\}-\boldsymbol{a}_{i}^{n-1}+2 \mathbf{a}_{i}^{n} .
$$

If the values of $\mathbf{a}^{0}$ and $\dot{\mathrm{a}}^{0}$ are prescribed as initial conditions, a special starting algorithm can be written by noting that

$$
\dot{\mathbf{a}}^{0}=\left(\mathbf{a}^{1}-\mathbf{a}^{-1}\right) / 2 \Delta t,
$$

and eliminating $\mathbf{a}^{-1}$ from equation (15b). 


\subsection{NUMERICAL EXAMPLES AND DISCUSSION}

In the present study the nine-noded quadrilateral isoparametric element was employed. The selective integration, that is, the $2 \times 2$ Gauss rule was used to integrate the shear energy terms and the $3 \times 3$ Gauss rule was used to integrate the bending and inertia terms. All the computations were carried in single precision on a CDC CYBER 180/840 Computer. Due to the biaxial symmetry of the problems discussed, only one quadrant of the plate was analyzed except for angle-ply plates which were analyzed by considering full plates with $4 \times 4$ mesh size (using zero initial conditions).

The following two sets of data were used in obtaining the numerical results: DATA 1: square plate $a=b=25 \mathrm{~cm}, h=5 \mathrm{~cm}, E_{1} / E_{2}=25, \rho=8 \times 10^{-6} \mathrm{~N} \mathrm{~s}^{2} / \mathrm{cm}^{4}, \nu=0 \cdot 25, E_{2}=$ $2 \cdot 1 \times 10^{6} \mathrm{~N} / \mathrm{cm}^{2}, G_{12}=G_{13}=G_{23}=0.5 E_{2}$; DATA 2: plate with $a=\sqrt{2}$ and $b=1, h=0 \cdot 2$, $\rho=1, \nu=0 \cdot 3, E_{2}=1 \cdot 0$ (non-dimensional).

In order to investigate the numerical convergence and accuracy of the transient behaviour, a simply supported isotropic and orthotropic $\left(0^{\circ} / 90^{\circ} / 90^{\circ} / 0^{\circ}\right)$ plate with a suddenly applied uniform pulse loading was analyzed with DATA 1 . The plate geometry, a typical finite element mesh, boundary conditions of the quarter model and applied loading are shown in Figure 2.

Tables 1 and 2 present centre deflection and normal stress for different meshes and time steps. From these tables it is found that the safe estimate of the critical time step lengths given by Leech [36], Tsui and Tong [37] and Hinton [38] are valid only for isotropic plates. The estimate of the critical time step is crucial in transient analysis of fibre reinforced composite thick plates. The estimate of the critical time step length of the transient solution of Mindlin plates given by Tsui and Tong [37] and Hinton [38]

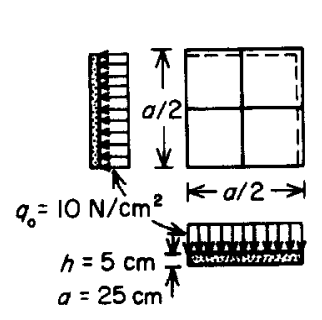

(a)

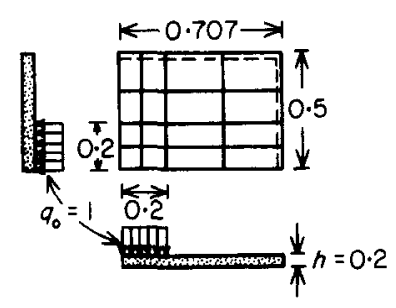

(b)

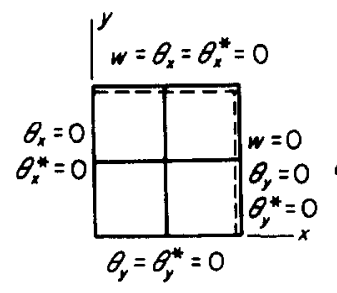

(c)

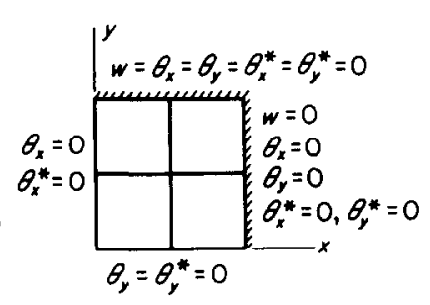

(d)
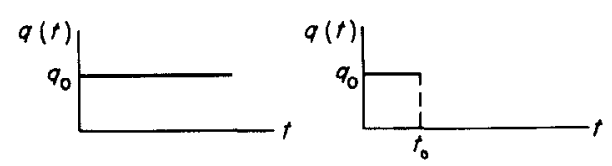

(e)

Figure 2. Geometry, bondary conditions and loading for DATA 1 and DATA 2. (a) DATA 1; (b) DATA 2; (c) simply supported boundary conditions; (d) clamped boundary conditions; (e) loading. 


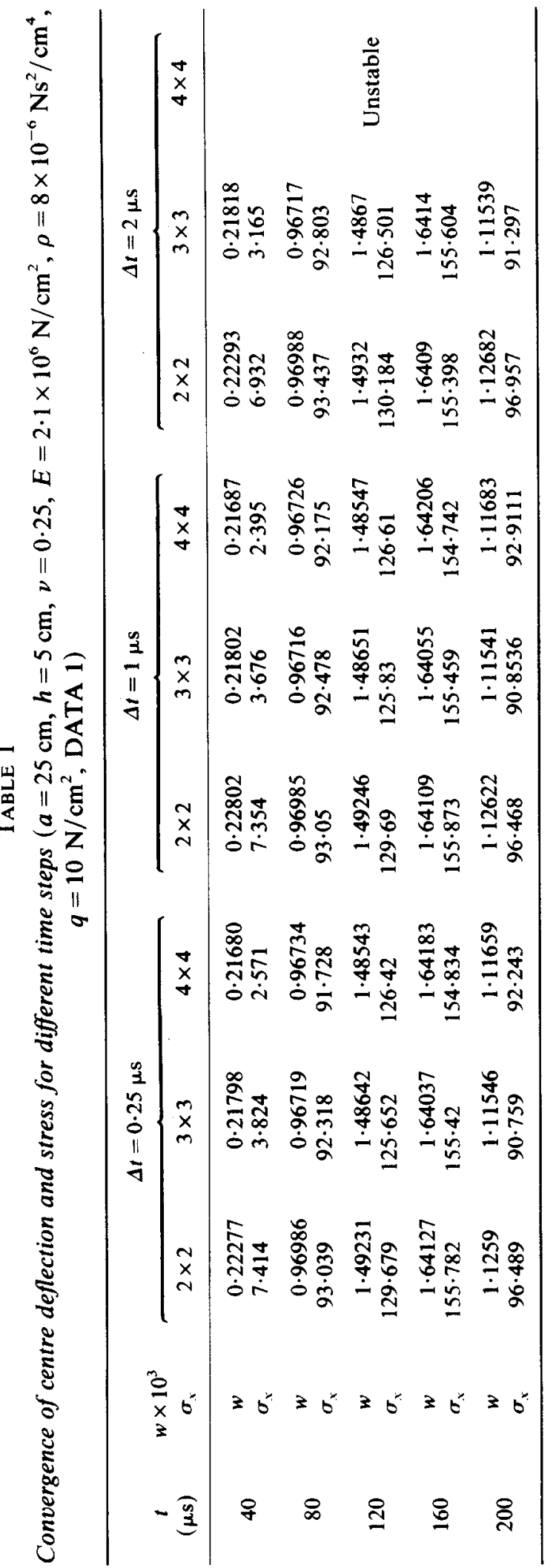




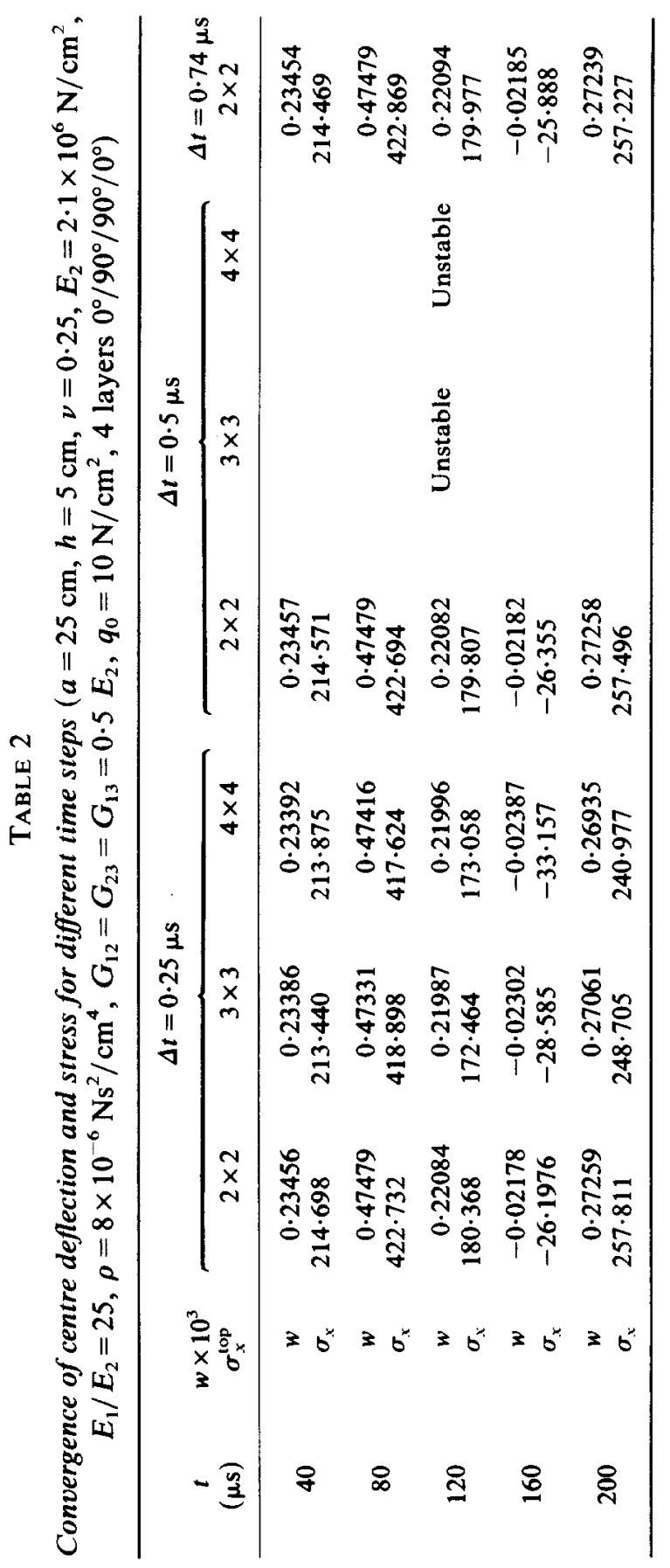


was used with minor modification in this study. The critical time step length is thus given as

$$
\Delta t=\Delta x\left[\frac{\mu\left(1-\nu^{2}\right) /\left(R E_{2}\right)}{2+0 \cdot 83(1-\nu)\left\{1+1 \cdot 5(\Delta x / h)^{2}\right\}}\right]^{1 / 2},
$$

where $R=E_{1} / E_{2}$, and $\Delta x$ is the smallest distance between adjacent nodes in any parabolic element used. $E_{1}$ and $E_{2}$ are the Young's moduli in the 1 and 2 directions respectively (see Figure 1).

In the present work the solution obtained with $\Delta t=0.25 \mu$ s was used for all the cases. The bound on the highest eigenvalue can be simply obtained by consideration of an individual element. This is established by an important theorem proposed by Irons [39] which proves that the highest system eigenvalue must always be less than the highest eigenvalues of the individual elements. This allows a very easy estimate of critical time steps.

Figure 3 shows a comparison of the plot of the centre deflection versus time with that of Akay [9]. The small phase difference between the present solution and that of Akay can be attributed to the difference in the formulations. The classical plate theory (i.e., not accounting for transverse shear strains) solution (from reference [35]) is also given in the figure to show the influence of the shear deformation on the centre deflection.

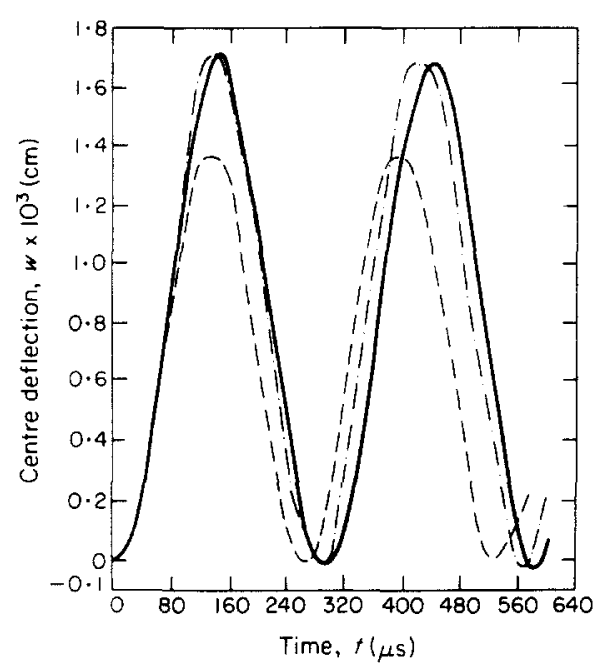

Figure 3. Centre deflection versus time for a simply supported square plate subjected to uniform pulse loading (DATA 1). - - Present, $\Delta t=0.25 \mu \mathrm{s} ;---$, mixed FEM [9], $\Delta t=5 \cdot 0 \mu \mathrm{s} ;---$, classical solution [35], $\Delta t=5 \cdot 0 \mu \mathrm{s}$.

In order to further validate the present theory, another problem for which the analytical solution exists has been solved. The problem consists of a simply supported rectangular plate (with DATA 2) subjected to a uniform pulse loading on a square (side $=0 \cdot 2 b$ ) area at the centre of the plate (see Figure 2). A non-uniform, $4 \times 4$ mesh of elements (with $\Delta t=0.02$ ) was employed. The problem was also solved analytically by Reismann and Lee [5], and by using the finite element method by Rock and Hinton [8]. A comparison of the non-dimensional centre deflection and non-dimensionalized bending moments $\left(\bar{M}_{y}\right)$ obtained by the present theory and by Reismann and Lee [5] is shown in Figures $4(a)$ and $4(b)$. The present finite element solution for the centre deflection is in excellent agreement with the thick plate solution. Since the bending moment in the present study 

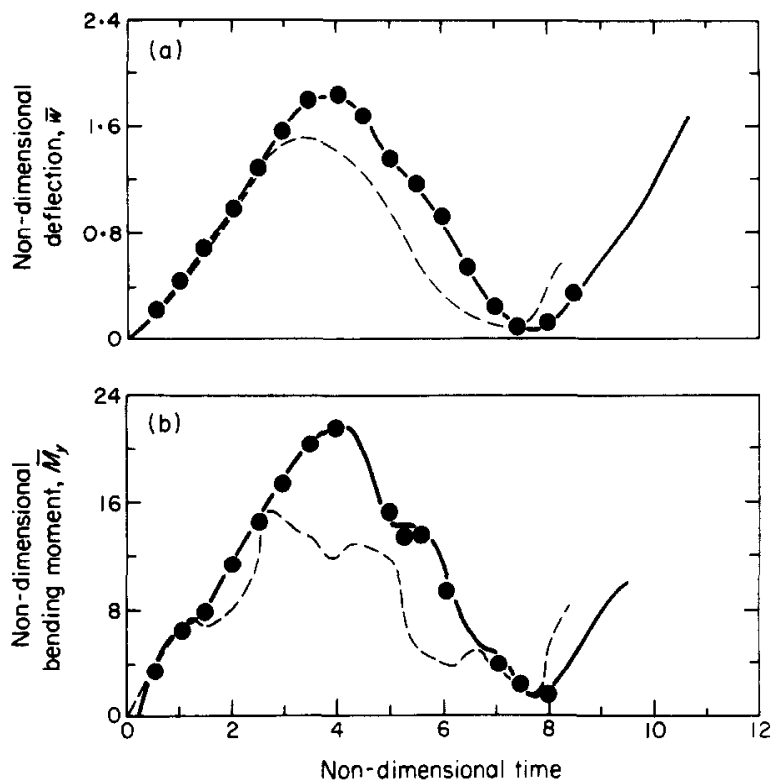

Figure 4. Non-dimensionalized centre deflection and bending moment versus time for simply supported, rectangular, isotropic plate under suddenly applied patch loading (DATA $2,4 \times 4, \Delta t=0.02, \bar{w}=w E a h / q_{0} b^{3}$, $\bar{M}_{y}=12 a M_{y} / q_{0} b^{2} h^{2}, T-t / b \sqrt{E / \rho}$ ). (a) Central deflection values; (b) central moment values. -...., Present FEM higher order theory; $\bullet$, analytical [5] thick plate theory; - - -, classical plate theory.
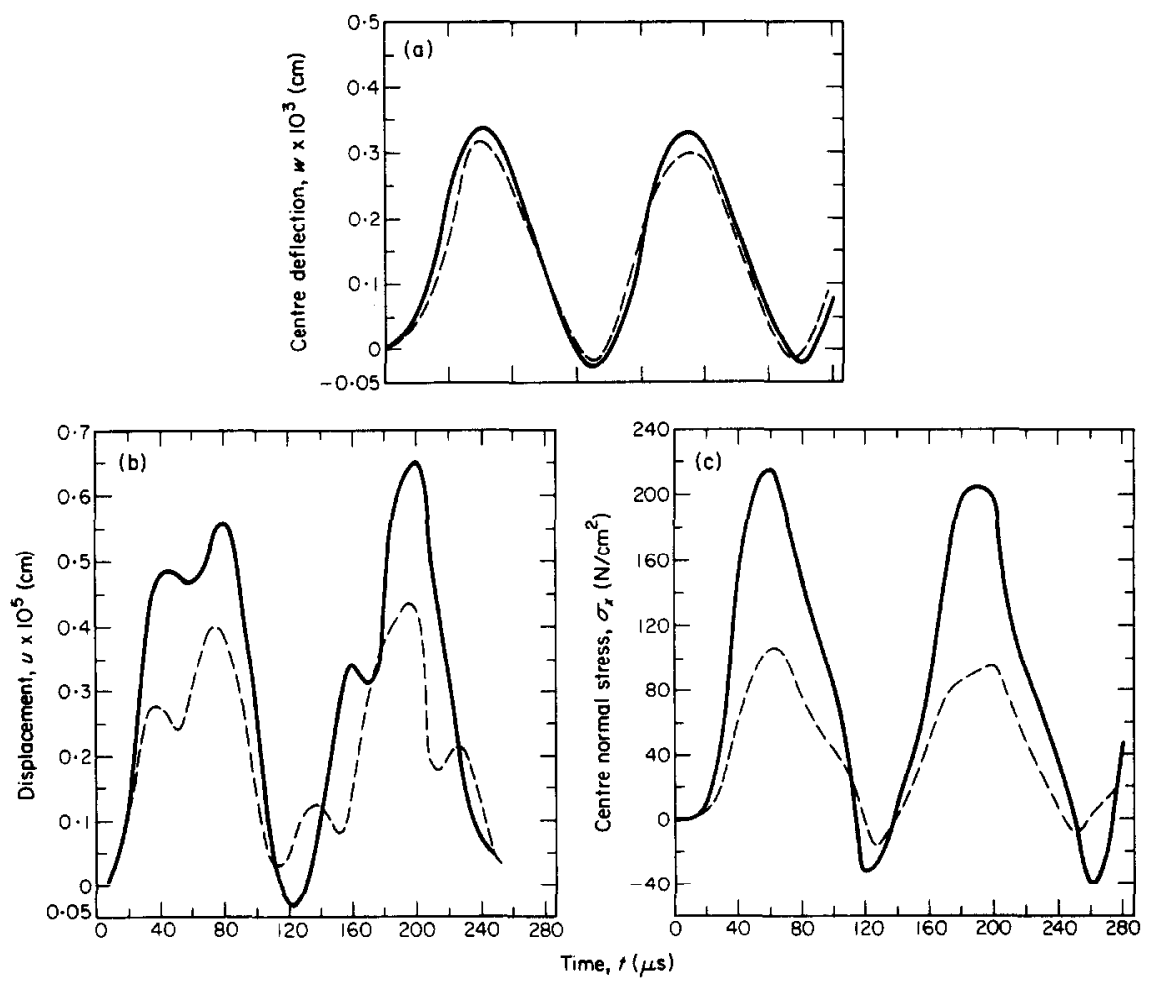

Figure 5. Centre deflection (a), axial displacement (b), and centre normal stress (c) layer $\left(0^{\circ} / 90^{\circ} / 0^{\circ}\right)$ clamped, square plate subjected to suddenly applied pulse loading. - - Present, $\Delta t=0.25 \mu \mathrm{s} ;---$, Mindlin [20], $\Delta t=5 \mu \mathrm{s}$. 

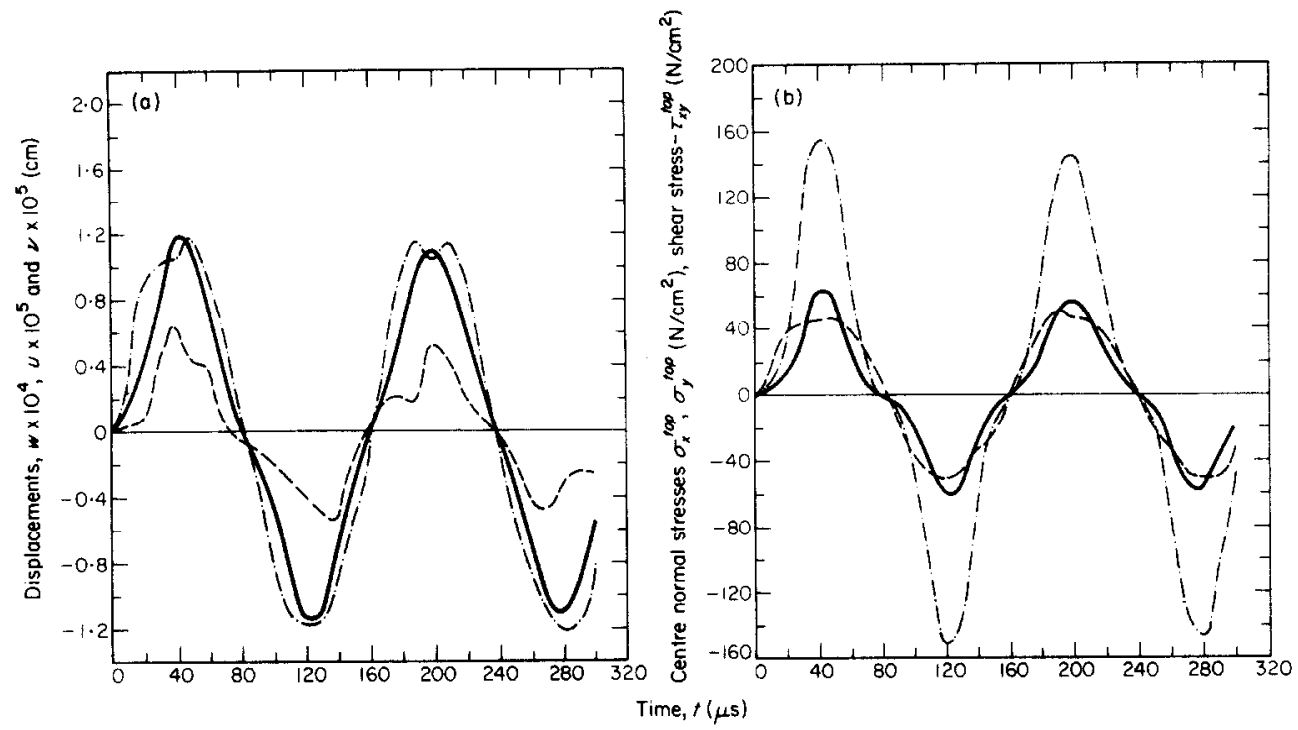

Figure 6. Centre deflection ( $\left.w_{0}\right)$ and axial displacements ( $u$ and $\left.v\right)(\mathrm{a})$, and stresses (b), versus time for a five layer $\left(60^{\circ} /-30^{\circ} / 0^{\circ} /-30^{\circ} / 60^{\circ}\right)$ simply supported plate under impulsive loading $\left(q=q_{0} \mathrm{H}\left(t-t_{0}\right)\right.$, DATA 1 , full plate analyzed with $4 \times 4$ mesh, $\left.\Delta t=5.0 \mu \mathrm{s}, t=10 \mu \mathrm{s}, q_{0}=10 \mathrm{~N} / \mathrm{cm}^{2}\right)$. For $(\mathrm{a}):-, \omega_{0} ;---u ;-\cdot-$, $v$; for $(b):-, \sigma_{x}^{\text {top }}$ of second layer; $-\cdots, \sigma_{y}^{\text {top }}$ of first layer;,$--- \tau_{x y}^{\text {top }}$ of first layer.

was calculated at the Gauss points, it is not expected to match exactly with that at the centre of the plate.

Next, results for plates of orthotropic materials and layered composite plates are discussed. Figure 5 shows the centre deflection $(w)$, the in-plane displacements $(u, v)$ at $x=6.25 \mathrm{~cm}$ and $y=9.375 \mathrm{~cm}$, and the normal stress for a three-layer $\left(0^{\circ} / 90^{\circ} / 0^{\circ}\right)$ clamped square plate (DATA 1) subjected to a suddenly applied pulse loading. A comparison with the Mindlin solution [20] is also shown in Figure 5. In the present displacement model, the in-plane displacements $u$ and $v$ at any point include higher order terms $z^{3} \theta_{x}^{*}$ and $z^{3} \theta_{y}^{*}$ which contribute to the cubic variation of planar deformations across the thickness. These additional contributions to the planar deformations are reflected in the results presented in Figure $5(\mathrm{~b})$. From this plot it is clear that Mindlin's plate theory predicts significantly lower values of displacements and stresses. Figure 6 shows the centre deflection, the in-plane displacements at $x=6.25 \mathrm{~cm}$ and $y=9.375 \mathrm{~cm}$, and the centre normal stress for a five layer $\left(60^{\circ} /-30^{\circ} / 0^{\circ} /-30^{\circ} / 60^{\circ}\right)$ simply supported square plate (DATA 1) subjected to an impulsive loading $\left(q_{0}=10 \mathrm{~N} / \mathrm{cm}^{2}, t_{0}=10 \mu \mathrm{s}\right)$ at the centre. Since no damping or internal friction is included in the present model the solutions do not decay with time.

\section{CONCLUSIONS}

The simple $C^{0}$ isoparametric formulation of an assumed higher-order displacement model employed herein is stable and accurate in predicting the transient response of composite plates. In contrast to the first-order shear deformation theory, the present higher order theory does not require a shear correction coefficient due to more realistic representation of the cross-sectional deformation. However, on the basis of the excellent agreement of the present results with analytical results for isotropic plates, it is fair to say that the theory is accurate in predicting the transient response of composite plates. 
Current and future investigations on this subject should be directed to forced vibration and impulse loadings in composite plates with damping included. Also, extension of these analyses to non-linear transient response of composite plates is awaiting attention.

\section{ACKNOWLEDGMENT}

Partial support of this research by the Aeronautics Research and Development Board, Ministry of Defence, Government of India through its Grant No. Aero/RD-134/100/84$85 / 362$ is gratefully acknowledged.

\section{REFERENCES}

1. S. G. LeKhnitskiI 1968 Anisotropic Plates. New York: Gordon and Breach. Second edition.

2. E. REISSNER and Y. STAVSKY 1961 Journal of Applied Mechanics 28, 402-408. Bending and stretching of certain types of heterogeneous aeolotropic elastic plates.

3. S. B. Dong, K. S. PISTER and R. L. TAYLOR 1962 Journal of Aerospace Science 29, 269-975. On the theory of laminated anisotropic shells and plates.

4. Y. STAVSKY 1961 Journal of Engineering Mechanics Division 97, 31-55. Bending and stretching of laminated aeolotropic plates.

5. H. REISMANN and Y. LEE 1969 Developments in Theoretical and Applied Mechanics, Vol. 4 (D. Frederick, editor), 3-18. New York: Pergamon. Forced motions of rectangular plates.

6. H. REISMANN 1968 Journal of Applied Mechanics 35, 510-515. Forced motion of elastic plates.

7. Y. LEE and H. REISMANN 1969 International Journal of Engineering Science 7, 93-113. Dynamics of rectangular plates.

8. T. ROCK and E. HINTON 1974 Earthquake Engineering and Structural Dynamics 3, 51-63. Free vibration and transient response of thick and thin plates using the finite element method.

9. H. U. AKAY 1980 Computers and Structures 11, 1-11. Dynamic large deflection analysis of plates using mixed finite elements.

10. F. C. MooN 1972 Journal of Composite Materials 6, 62-79. Wave surface due to impact on anisotropic plates.

11. F. C. MOON 1973 Journal of Applied Mechanics 40, 485-490. One dimensional transient waves in anisotropic plates.

12. T. S. CHOw 1971 Journal of Composite Materials 5, 306-319. On the propagation of flexural waves in an orthotropic laminated plate and its response to an impulsive load.

13. A. S. D. WANG, P. C. CHOU and J. L. ROSE 1972 American Institute of Aeronautics and Astronautics Journal 10, 1088-1090. Strongly coupled stress waves in heterogeneous plates.

14. C. T. SUN 1973 Journal of Composite Materials 7, 366-382. Propagation of shock waves in anisotropic composite plates.

15. C. T. SUN and J. M. WHITNEy 1974 Journal of the Acoustical Society of America 55(5), 1003-1008. Forced vibrations of laminated composite plates in cylindrical bending.

16. C. T. SUN and J. M. WHITNEY 1975 American Institute of Aeronautics and Astronautics Journal 13(10), 1259-1260. Dynamic response of laminated composite plates.

17. J. M. WHITNEY and C. T. SUN 1977 Journal of the Acoustical Society of America 61(1), 101-104. Transient response of laminated composite plates subjected to transverse dynamic loading.

18. R. D. MindLin and L. F. GoOdman 1950 Journal of Applied Mechanics 17, 377-380. Beam vibrations with time-dependent boundary conditions.

19. J. N. REDDY 1982 Journal of Applied Mechanics 49, 403-408. On the solutions to forced motions of rectangular composite plates.

20. J. N. REDDY 1983 International Journal for Numerical Methods in Engineering 19, 237-255. Dynamic (Transient) analysis of layered anisotropic composite-material plates.

21. M. V. V. MURTHY 1981 NASA Technical Paper-1903. An improved transverse shear deformation theory for laminated anisotropic plates.

22. N. D. PHAN and J. N. REDDY 1985 International Journal for Numerical Methods in Engineering 21, 2201-2219. Analysis of laminated composite plates using a higher-order shear deformation theory.

23. K. H. Lo, R. M. Christensen and E. M. Wu 1977 Journal of Applied Mechanics 44, 669-676. A higher-order theory of plate deformation-Part 2: Laminated plates. 
24. T. KANT 1982 Computer Methods in Applied Mechanics and Engineering 31, 1-18. Numerical analysis of thick plates.

25. T. KANT, D. R. J. OWEN and O. C. ZIENKIEWICZ 1982 Computers and Structures 15, 177-183. $A$ refined higher-order $C^{0}$ plate bending element.

26. T. KANT and B. N. PANDYA 1988 Proceedings of the International Conference on Composite Materials and Structures, 6-9 January, 1988, Madras. Finite element stress analysis of unsymmetrically laminated composite plates based on a refined higher-order theory.

27. T. KANT and B. N. PANDYA 1988 Journal of Composite Structures 9(3), 215-246. A simple finite element formulation of a higher-order theory for unsymmetrically laminated plates.

28. T. KANT and B. N. PANDYA 1987 Symposium on Delaminations in Composites, 19-20 March, 1987, I. I. Sc. Bangalore, India. Finite element evaluation of interlaminar stresses based on first and higher-order theories.

29. B. N. PANDYA and T. KANT 1987 Mechanics-Research Communications 14(2), 107-113. A consistent refined theory for flexure of a symmetric laminate.

30. B. N. PANDYA AND T. KANT 1988 Computer Methods in Applied Mechanics and Engineering 66, 173-198. Flexural analysis of laminated composite using refined higher-order $C^{0}$ plate bending elements.

31. B. N. PANDYA and T. KANT 1987 Computers and Structures 28, 119-133. A refined higher-order generally orthotropic $\mathrm{C}^{0}$ plate bending element.

32. R. M. JONES 1975 Mechanics of Composite Materials. New York: McGraw-Hill.

33. A. K. GUPTA and A. MOHRAZ 1972 International Journal of Numerical Methods in Engineering 5, 83-92. A method of computing numerically integrated stiffness matrices.

34. E. HINTON, T. ROCK and O. C. ZIENKIEWICZ 1976 Earthquake Engineering and Structural Dynamics 4, 245-249. A note on mass lumping and related processes in the finite element method.

35. E. VolterRa and E. C. ZACHMANOGloU 1965 Dynamics of Vibrations. Ohio: Merrill Columbus.

36. J. W. LEECH 1965 American Institute of Aeronautics and Astronautics Journal 3(9), 1772-1773. Stability of finite difference equations for transient response of flat plates.

37. T. Y. TSUI and P. ToNG 1971 American Institute of Aeronautics and Astronautics Journul 9, 2062-2063. Stability of transient solution of moderately thick plates by finite difference methods.

38. E. HINTON 1976 Journal of Sound and Vibration 46(4) 465-472. The dynamic transient analysis of axisymmetric circular plates by the finite element method.

39. B. M. IRONS and S. AHMAD 1980 Finite Element Techniques. Chichester: Ellis Horwood. 